\title{
Las personas con discapacidad intelectual como investigadoras. Debates, retos y posibilidades de la investigación inclusiva
}

People with intellectual disabilities as researchers. Discussions,
challenges and possibilities of inclusive research

\author{
Judit Fullana Noell
}

Universidad de Girona

judit.fullana@udg.edu(ESPAÑA)

Maria Pallisera Díaz

maria.pallisera@udg.edu(ESPAÑA)

Montserrat Vilà Suñé

montserrat.vila@udg.edu(ESPAÑA)

Carolina Puyalto Rovira

carolina.puyalto@udg.edu(ESPAÑA)

Recibido: 19.04 .2015

Aceptado: 04.01.2016

\section{RESUMEN}

En las últimas dos décadas se ha desarrollado, especialmente en el contexto anglosajón, una enfoque de investigación social y educativa denominado "investigación inclusiva" (Walmsley y Johnson, 2003) que promueve la participación de las personas con discapacidad intelectual en la investigación, bien sea como asesoras, como co-investigadoras, o bien como líderes y promotoras de investigaciones sobre temas que les afectan. Este desarrollo se ha producido a raíz de la progresiva implementación del modelo social en el análisis de la discapacidad y de la constitución de grupos de autodefensa que ganan protagonismo tras la publicación de la Convención de Naciones Unidas sobre los Derechos de las personas con Discapacidad (2006). En este artículo presentamos los principales 
debates metodológicos que ha generado la inclusión de las personas con discapacidad intelectual en los procesos de toma de decisiones sobre la investigación, y planteamos los principales debates y retos, sobre todo desde el punto de vista metodológico, que plantea dicha inclusión. Los principales debates tienen relación con la participación de las personas con discapacidad intelectual en la investigación y los apoyos necesarios, su representatividad en las distintas fases y la equidad de las relaciones entre investigadores con y sin discapacidad. Los retos, que derivan de estos debates, se sitúan en torno a cómo proporciona oportunidades para participar, para estar representados en las distintas fases y en torno al compromiso ético de los investigadores sin discapacidad para asegurar la participación. Basamos nuestro análisis en la revisión de la literatura sobre los procesos de investigación inclusiva y en la reflexión fruto de nuestra propia experiencia como investigadoras que hemos desarrollado procesos inclusivos de investigación en los últimos años.

\title{
PALABRAS CLAVE
}

Investigación inclusiva, Discapacidad intelectual, Investigación participativa, Metodología de investigación, Papel del investigador.

\begin{abstract}
In the last two decades, an approach to social an educational research, called, inclusive research (Walmsley and Johnson, 2003), has been developed, especially in the Anglo-Saxon context. This approach promotes the participation of people with intellectual disabilities in research, either as advisors, as coresearchers, or as leaders and promoters of research on issues that affect them. This development has occurred as a result of the progressive implementation of the social model of disability analysis and the emergence of self-advocacy groups who have gained prominence following the release of the UN Convention on the Rights of Persons with Disabilities (2006). This article introduces some methodological debates generated by the inclusion of people with intellectual disabilities in decision-making processes on research, and proposes the main discussions and challenges that inclusion posed, especially from the methodological point of view. The main debates are related to the participation of people with intellectual disabilities in research and their support needs, their representation in the research phases and equity relationships between researchers with and without disabilities. The methodological challenges arising from these discussions are around how to provide opportunities to participate, to be represented at different research stages and about ethical commitment of researchers without disabilities to ensure participation. Our analysis relies on inclusive research literature review and on the reflection about our own experience as researchers who have developed inclusive processes of research in recent years.
\end{abstract}




\section{KEY WORDS}

Inclusive Research, Intellectual Disability, Participatory Research, Research Methodology, Role of the researcher.

\section{INTRODUCCIÓN}

La Convención Internacional sobre los Derechos de las Personas con Discapacidad (Naciones Unidas, 2006) establece que las personas con discapacidad son titulares de derechos y que los poderes públicos están obligados a garantizar el ejercicio pleno y efectivo de estos derechos. Orientada por los principios de participación e inclusión, no discriminación e igualdad de oportunidades, la Convención establece claramente los derechos a la inclusión educativa y laboral, a la vida independiente y a la participación en la comunidad, entre otros. Sin embargo, aunque en las últimas décadas, en los países occidentales se ha producido un considerable avance en la inclusión social de las personas con discapacidad intelectual, la investigación demuestra que, en la práctica, estas personas tienen muchas dificultades para poder ejercer plenamente sus derechos. Estudios realizados en distintos países ponen de manifiesto que las personas con discapacidad intelectual encuentran obstáculos para obtener una formación profesional que les permita afrontar la vida laboral con los recursos adecuados (Beresford, 2004; Clarke et al., 2011; Pallisera et al., 2014a); para participar en la comunidad y para llevar a cabo sus proyectos de vida independiente y su proceso de emancipación (Emerson y Ramcharan, 2010; European Commission, 2009; FRA-European Union Agency for Fundamental Rights, 2013). Además, se señala que en muchas ocasiones no se sienten satisfechas con los servicios que reciben (Deguara et al., 2012; McConkey et al., 2004).

La voz de las personas con discapacidad es fundamental para conocer qué es lo que ellas desean en sus vidas, cómo valoran los servicios que se les ofrecen y para poder planificar y desarrollar los apoyos necesarios que garanticen su participación en todos los ámbitos de la comunidad (Abbott y McConkey, 2006). La investigación social y educativa, entendida como una actividad al servicio de las personas y de la sociedad, debe contribuir a dar voz a las personas con discapacidad. Desde esta perspectiva, considerar su inclusión en los procesos de investigación social y educativa es una condición necesaria para responder de forma efectiva a las necesidades reales de estas personas, contribuir a la mejora de sus condiciones de vida y hacer efectivos sus derechos.

Desde finales de la década de los 90, especialmente en el contexto anglosajón, se ha avanzado en la inclusión de la perspectiva de las personas con discapacidad en la investigación. Así, en contraposición a las prácticas habituales en la investigación sobre discapacidad, el modelo de investigación inclusiva (Walmsley, 2001, 2004 y Walmsley y Johnson, 2003) defiende que las personas con experiencias personales relevantes en relación al tema 
investigado deben ser incluidas en el estudio, y debe facilitarse su participación en todas las fases del mismo. Esta participación supone un valor añadido porque enriquece el proceso de investigación a través la inclusión de las perspectivas y experiencias de las personas con discapacidad, aumenta las posibilidades de incidir en el cambio político y social a través de la defensa de sus derechos y proporciona apoyo al desarrollo de sus proyectos de vida (Johnson, Minogue y Hopkins, 2014; Koenig, 2011; O’Brien, McConkey y García-Iriarte, 2014). Se considera que fomentar la participación de las personas con discapacidad en la investigación sobre temas que les afectan constituye una responsabilidad ética de los investigadores comprometidos con la mejora de la inclusión de las personas con discapacidad en la sociedad.

En este contexto, este artículo tiene como objetivo presentar los principales debates que se han generado hasta estos momentos con respecto a la inclusión de las personas con discapacidad intelectual en los procesos de investigación. Para ello, partimos del análisis de la literatura sobre los procesos de investigación inclusiva. Tomando como base esta revisión y la reflexión sobre nuestra propia experiencia como investigadoras que hemos desarrollado procesos inclusivos en la investigación con personas con discapacidad intelectual en los últimos años, se plantean algunos retos y posibilidades de dicha inclusión.

\section{LA PARTICIPACIÓN DE LAS PERSONAS CON DISCAPACIDAD INTELECTUAL EN LOS PROCESOS DE INVESTIGACIÓN. EL DESARROLLO DE INVESTIGACIONES INCLUSIVAS}

El término investigación inclusiva hace referencia a un planteamiento de investigación en el que las personas con discapacidad intelectual tienen un papel activo en los estudios sobre cuestiones que son de su interés, ya sea como financiadoras, asesoras, investigadoras, o difusoras de la investigación (TuffreyWijne y Butler, 2009). Walmsley y Johnson (2003:10) definen la investigación inclusiva como un rango de enfoques de investigación que tradicionalmente ha sido adjetivada como 'participativa', 'acción' o 'emancipadora'. Stack y McDonald (2014) sitúan la investigación inclusiva como una rama de la investigación acción que, junto con la investigación acción participativa, la investigación participativa basada en la comunidad y la investigación emancipadora comparten los siguiente principios: cooperación recíproca igualitaria entre miembros de comunidades explotadas y oprimidas y miembros de la academia, fomento de experiencias de co-aprendizaje partiendo de los temas que preocupan a la comunidad, empoderamiento de los miembros de la comunidad de forma que puedan ejercer mayor control sobre sus vidas, y equilibrio entre investigación y acción comunitaria centrada en el cambio en la persona y en el sistema.

Dentro de este contexto, el aspecto particular de la investigación inclusiva es que los participantes son personas con discapacidad y bajo este enfoque se han desarrollado investigaciones con distintas formas y niveles de implicación de las 
personas con discapacidad intelectual.

\subsection{Investigación inclusiva, modelo social de la discapacidad y derechos}

La perspectiva inclusiva en la investigación se relaciona con la progresiva implementación del modelo social en el análisis de la discapacidad (Nind, 2011). Este modelo irrumpe con fuerza en Europa en los años 80 y se desarrolla en los 90 como enfoque opuesto al modelo individual o médico predominante hasta ese momento. Así, se produce la transición desde una perspectiva que ve a las personas con discapacidad como personas incapaces de tomar decisiones, necesitadas de protección y de personas que hablen en su nombre, a una nueva manera de entender la discapacidad. La nueva perspectiva considera que estas personas tienen capacidad de autoconfianza, de hablar en beneficio propio y de defender sus propios intereses, de tomar decisiones y de tener una voz por sí mismas (William y Shoultz, 1982). La progresiva constitución de grupos de autodefensa (self-advocacy) contribuye de forma significativa a este cambio de perspectiva (Walmsley y The Central England People First History Project Team, 2014).

A lo largo de los últimos 20 años ha ido aumentando la importancia de la investigación inclusiva en el campo de los estudios sobre discapacidad, especialmente en el ámbito anglosajón, fruto del desarrollo del modelo social de la discapacidad y del movimiento "Nothing about us without us" que impulsa los movimientos de auto-defensa de los derechos de la personas con discapacidad (Strnadová y Cumming, 2014). Actualmente existe bastante acuerdo en que la investigación inclusiva deber incluir los principios siguientes (Walmsley y Johnson, 2003).

- El problema de investigación o el tema debe pertenecer a las personas con discapacidad intelectual, aunque no sean ellas las iniciadoras del proceso.

- Debe ser de interés para las personas con discapacidad intelectual y los investigadores sin discapacidad deben apoyar a las personas con discapacidad.

- Debe ser colaborativa: las personas con discapacidad intelectual deben implicarse en el proceso con la ayuda de personas sin discapacidad.

- Las personas con discapacidad deberían poder ejercer cierto control sobre el proceso y los resultados de la investigación.

- La pregunta de investigación, el proceso y los informes deben ser accesibles a las personas con discapacidad.

La investigación participativa y la investigación emancipadora mantienen conexiones importantes con la investigación inclusiva. Algunos autores han tratado de diferenciar ambos enfoques, aduciendo que el enfoque emancipador se produce cuando la iniciativa y el foco de la investigación es propuesto por 
las personas con discapacidad y ellas controlan la investigación, mientras que el enfoque participativo pone mayor énfasis en la representación de las personas con discapacidad en todos los estadios del proceso de investigación, estableciendo alianzas con investigadores sin discapacidad (Boland, Daly y Staines, 2008; Chappell, 2000). En la práctica, sin embargo, el término "investigación inclusiva" engloba investigaciones desarrolladas desde ambas perspectivas.

\subsection{Modelos de participación en la investigación inclusiva}

Según la forma de participación de las personas con discapacidad intelectual pueden identificarse tres enfoques principales: investigaciones en las que estas personas actúan como asesoras, investigaciones en la que actúan como líderes e investigaciones colaborativas (Bigby, Frawley y Ramcharan, 2014a).

La actuación de personas con discapacidad intelectual como asesoras de la investigación puede incluir todos o algunos de los siguientes elementos: establecer prioridades, decidir sobre la financiación, asesorar a los equipos sobre distintas fases de la investigación como su diseño, las estrategias para seleccionar a los participantes, la recogida y el análisis de datos o los métodos de difusión. Ejemplos de este tipo de investigación son los trabajos de Boland, et al. (2008), Brooks y Davies (2008), Keyes y Brandon (2011), Kidney y McDonald (20149, Johnson, Minogue y Hopkins (2014) y Young y Chesson (2007), entre otras (tabla 1). Las personas con discapacidad intelectual participan como asesoras, a veces en comités formados por representantes de distintos ámbitos (servicios que atienden a personas con discapacidad, familiares, profesionales de distintos ámbitos, e investigadores de la universidad), en la definición de temas de investigación, la elaboración de instrumentos de recogida de datos, el análisis de datos, y la difusión de los resultados.

La participación de personas con discapacidad intelectual como líderes de la investigación implica que éstas toman el control y deciden iniciar y llevar a cabo sus propias investigaciones sobre temas que son importantes para ellas. Ejemplos de este planteamiento los encontramos en Mitchell (2012) y Deguara (2012) (tabla 1). Este tipo de participación se ha llevado a cabo generalmente mediante procesos de investigación acción, utilizando metodologías participativas de modo que las personas con discapacidad, a través de un proceso de empoderamiento, lleguen a ser sujetos activos de su propia indagación y, además, impulsen el cambio y la transformación social. Este enfoque es menos frecuente, desarrollándose por lo general en el contexto de organizaciones de autodefensa que muestran interés por determinados temas que afectan a la vida de las personas con discapacidad intelectual y buscan la colaboración con investigadores sin discapacidad para llevar a cabo sus iniciativas.

El tercer enfoque sitúa a las personas con discapacidad intelectual en grupos de investigación colaborativos, que a veces han sido denominados grupos de co- 
investigación (Walmsley y Johnson, 2003). Para Bigby, Frawley y Ramcharan (2014a), la investigación colaborativa se refiere a colaboraciones en las que personas con y sin discapacidad trabajan juntas sobre objetivos compartidos, sus aportaciones son valoradas equitativamente y tanto unas como otras ostentan el mismo grado de poder o control sobre la investigación. Los investigadores sin discapacidad intelectual no actúan simplemente como ayudantes sino que combinan las habilidades de los investigadores académicos con habilidades de las personas con discapacidad intelectual para generar nuevos conocimientos que ninguno de los dos grupos podría conseguir por separado. Como ejemplos de este enfoque de investigación podemos citar a Abell et al. (2007), Bigby et al (2014b), Haigh et al. (2013), Ollerton y Horsfall (2013), Turk et al. (2012) y White y Morgan (2012) (tabla 1).

\subsection{Investigación inclusiva en el contexto español}

Todo este movimiento desarrollado en el Reino Unido, Irlanda y en otros países de influencia anglosajona, como E.E.U.U. y Australia, ha tenido escasa influencia en España. A pesar de la progresiva asunción del modelo social de la discapacidad y de la ratificación en el 2008 de la Convención Internacional sobre los Derechos de las Personas con Discapacidad (ONU, 2006), la participación de las personas con discapacidad intelectual en las investigaciones representa todavía un desafío en nuestro contexto si bien algunas voces ya señalan la necesidad de desarrollar experiencias investigadoras inclusivas (González Luna, 2013; Haya, Rojas y Lázaro, 2014; Susinos y Parrilla, 2008, 2013). Entre las experiencias documentadas desarrolladas de forma inclusiva, destacamos la de Cortés (2012), en la que el grupo de investigación Nosotros también investigamos, integrado por cinco personas con discapacidad intelectual y una persona de apoyo, realiza un estudio sobre la participación de los usuarios con discapacidad de un centro ocupacional. Por otro lado, desde nuestro grupo de investigación, llevamos a cabo una investigación sobre la transición a la vida adulta de jóvenes con discapacidad intelectual ${ }^{1}$ con la colaboración de un comité asesor integrado

${ }^{1}$ Proyecto La transición a la edad adulta y vida activa de jóvenes con discapacidad intelectual desde una perspectiva inclusiva: identificación de problemas, buenas prácticas y propuestas de un plan de mejora (EDU2011-22945). 


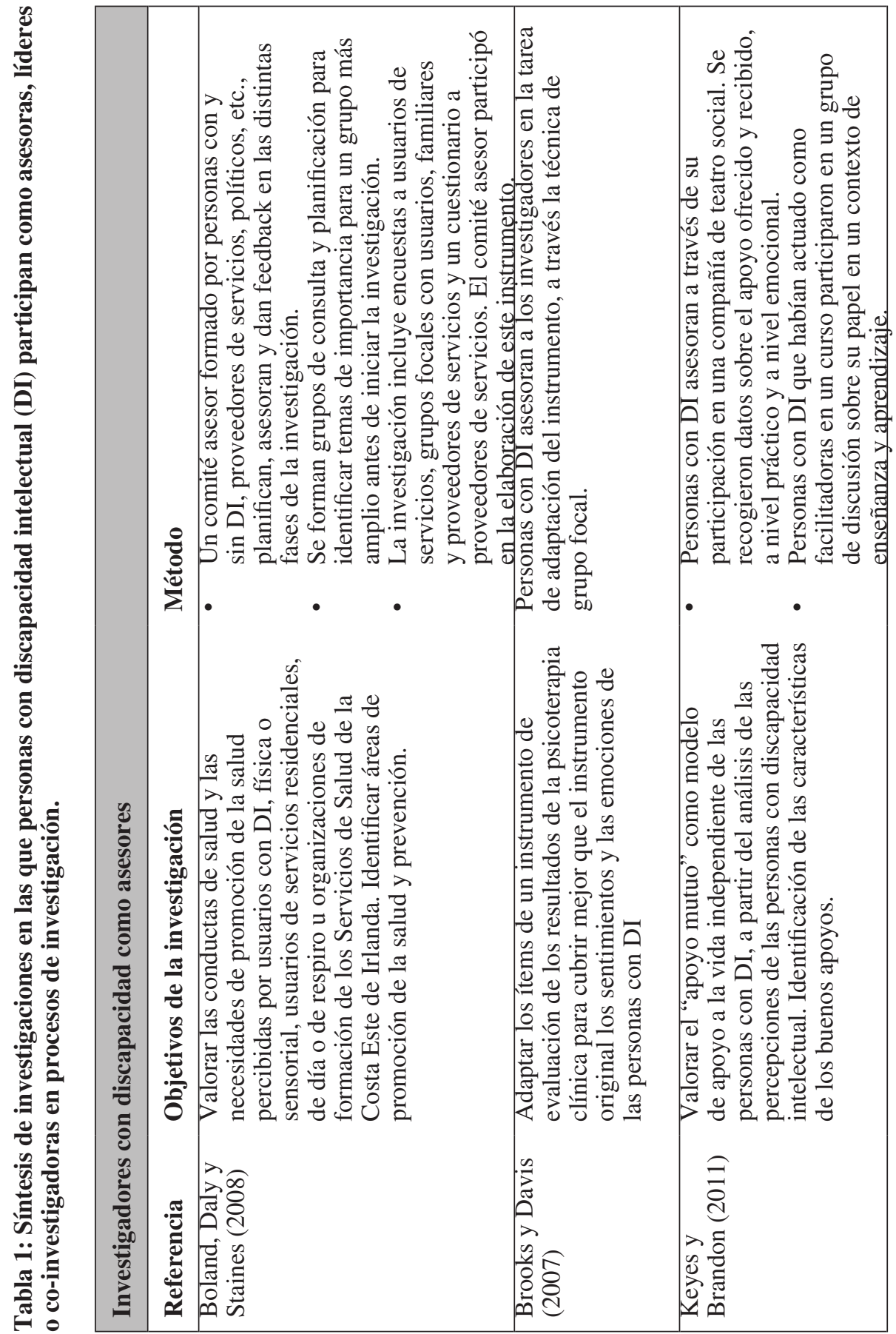

EMPIRIA. Revista de Metodología de Ciencias Sociales. N. ${ }^{\circ} 33$, enero-abril, 2016, pp. 111-138. ISSN: 1139-5737, DOI/empiria.33.2016.15866 


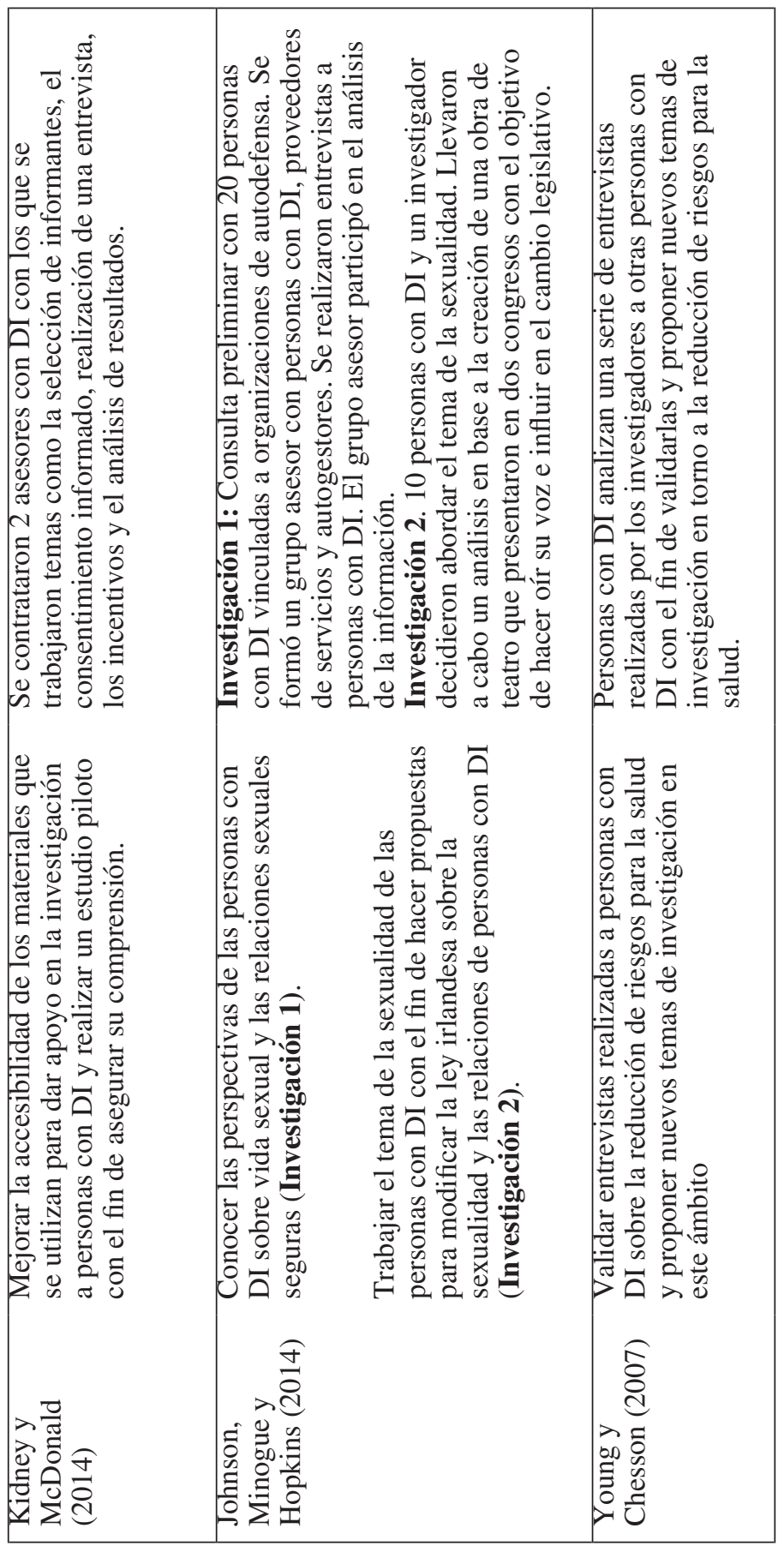

EMPIRIA. Revista de Metodología de Ciencias Sociales. N. ${ }^{\circ} 33$, enero-abril, 2016, pp. 111-138. ISSN: 1139-5737, DOI/empiria. 33.2016.15866 


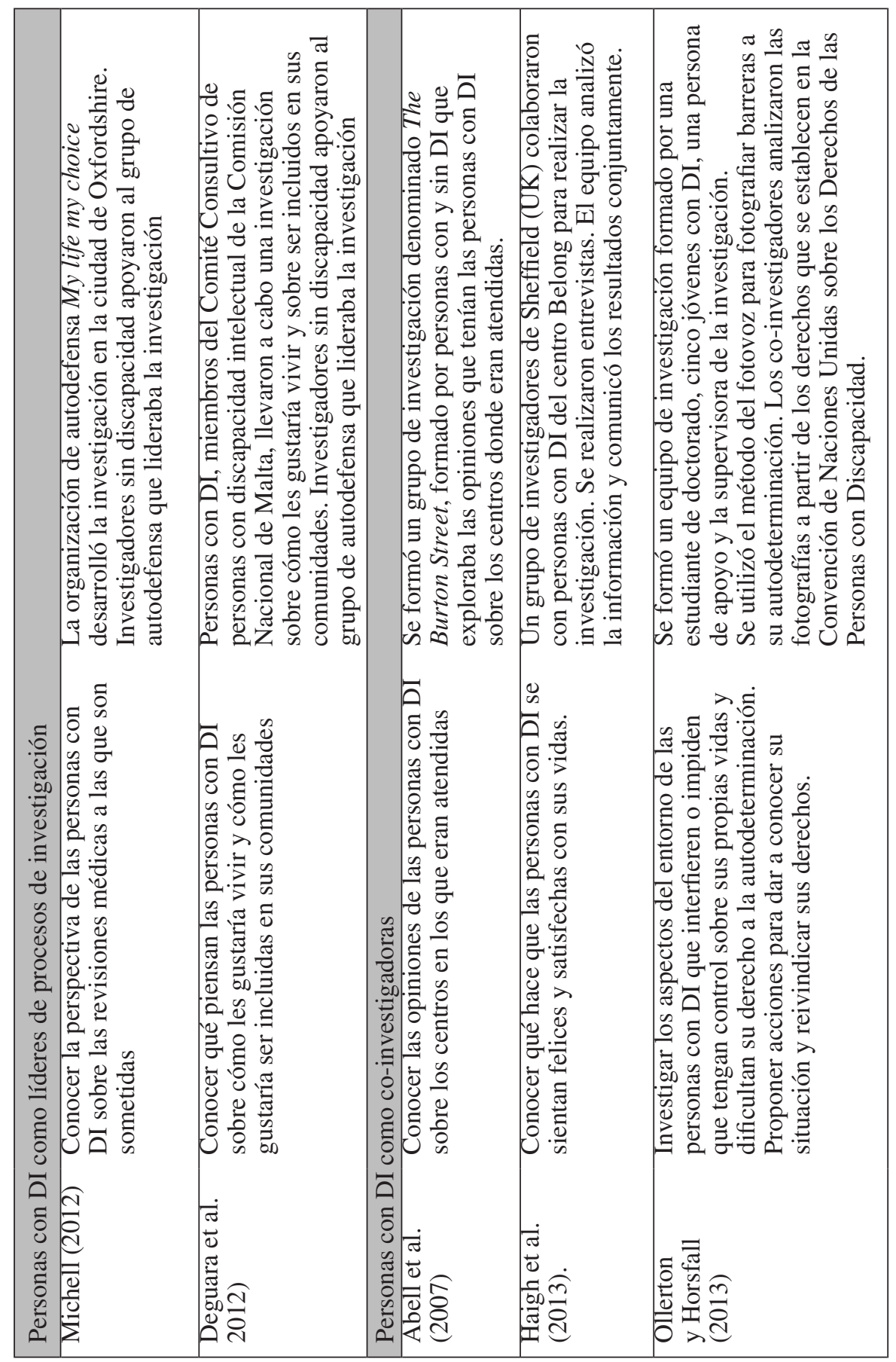

EMPIRIA. Revista de Metodología de Ciencias Sociales. N. ${ }^{\circ} 33$, enero-abril, 2016, pp. 111-138. ISSN: 1139-5737, DOI/empiria.33.2016.15866 


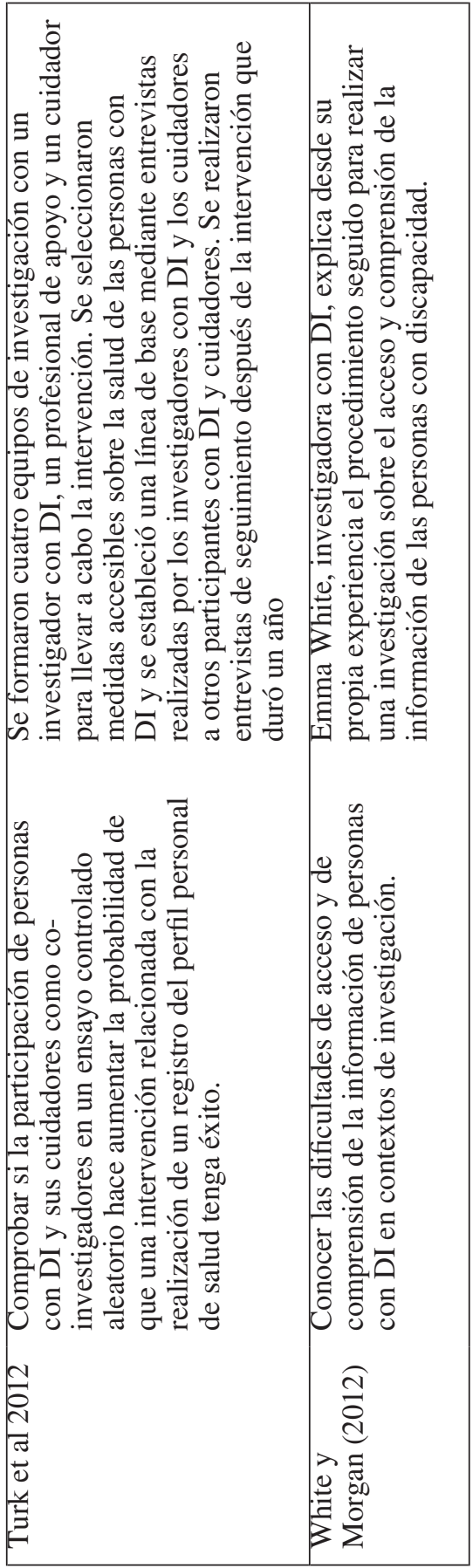

EMPIRIA. Revista de Metodología de Ciencias Sociales. N. ${ }^{\circ} 33$, enero-abril, 2016, pp. 111-138. ISSN: 1139-5737, DOI/empiria. 33.2016.15866 
por personas con discapacidad intelectual (tabla 2). Durante el primer año (2012-2013), diez personas integraron el comité con el objetivo de asesorar al equipo de investigadores en el trabajo vinculado al objetivo 2 , correspondiente al desarrollo de un estudio de casos con jóvenes con discapacidad intelectual. El comité asesor colaboró en el diseño de los instrumentos de recogida de datos y en el análisis, interpretación y difusión de los mismos (Pallisera et al., 2013a).

Tabla 2: Objetivos, método y funciones del comité asesor en una investigación sobre transición de jóvenes con discapacidad intelectual (DI) a la vida adulta.

\section{Título: La transición a la vida adulta de jóvenes con discapacidad intelectual. Diagnóstico y propuestas de mejora}

\begin{tabular}{|ll|}
\hline Objetivos & Método \\
\hline $\begin{array}{l}\text { Realizar un } \\
\text { diagnóstico }\end{array}$ & $\begin{array}{l}\text { Estrevistas a profesionales } \\
\text { formados por profesionales del ámbito escolar y profesionales }\end{array}$ \\
en & del ámbito postescolar \\
profundidad & \\
del & \\
sistema de & \\
dispositivos & \\
y servicios & \\
relacionados & \\
con la & \\
transición & \\
\hline
\end{tabular}




\begin{tabular}{|c|c|c|c|}
\hline \multirow[b]{2}{*}{$\begin{array}{l}\text { 2. Identificar } \\
\text { los } \\
\text { principales } \\
\text { problemas } \\
\text { y conocer } \\
\text { los factores } \\
\text { facilitadores } \\
\text { de la } \\
\text { transición, } \\
\text { desde la } \\
\text { propia } \\
\text { experiencia } \\
\text { de los } \\
\text { jóvenes con } \\
\text { discapacidad. }\end{array}$} & \multirow[b]{2}{*}{$\begin{array}{l}\text { Estudio } \\
\text { de caso de } \\
8 \text { jóvenes } \\
\text { con DI } \\
\text { participantes } \\
\text { en un } \\
\text { programa de } \\
\text { formación } \\
\text { para la } \\
\text { transición a la } \\
\text { vida adulta. } \\
\text { Grupos } \\
\text { focales con } \\
\text { jóvenes con } \\
\text { DI }\end{array}$} & \multicolumn{2}{|c|}{ Comité asesor } \\
\hline & & $\begin{array}{l}\text { Funciones } \\
\text { Asesorar en la } \\
\text { elaboración de } \\
\text { los instrumentos } \\
\text { (entrevistas y } \\
\text { grupos focales). } \\
\text { Participar en } \\
\text { el análisis e } \\
\text { interpretación } \\
\text { de los datos } \\
\text { procedentes de } \\
\text { las entrevistas y } \\
\text { grupos focales. } \\
\\
\text { Participar en la } \\
\text { elaboración de } \\
\text { materiales de } \\
\text { difusión. }\end{array}$ & $\begin{array}{l}\text { Procedimiento } \\
\text { Reunión 1: Discusión } \\
\text { sobre los temas } \\
\text { relacionados con } \\
\text { el tránsito a la vida } \\
\text { adulta, identificando los } \\
\text { principales problemas, } \\
\text { así como los apoyos } \\
\text { que pueden facilitar la } \\
\text { transición. } \\
\text { Reunión 2: Análisis } \\
\text { e interpretación de los } \\
\text { datos procedentes de las } \\
\text { entrevistas. } \\
\text { Reunión 3: Análisis e } \\
\text { interpretación de datos de } \\
\text { los grupos focales. } \\
\text { Reunión 4: Análisis } \\
\text { de las dificultades y } \\
\text { apoyos en el proceso de } \\
\text { transición a la vida adulta } \\
\text { a través de fotografías } \\
\text { realizadas por los propios } \\
\text { asesores. } \\
\text { Reunión 5: Elaboración } \\
\text { de una presentación } \\
\text { a un congreso con el } \\
\text { objetivo de explicar el } \\
\text { proceso realizado y los } \\
\text { aprendizajes adquiridos. } \\
\text { Reunión 6: Análisis del } \\
\text { papel del trabajo en el } \\
\text { desarrollo de proyectos } \\
\text { de vida. }\end{array}$ \\
\hline $\begin{array}{l}\text { 3. Proponer } \\
\text { acciones o } \\
\text { estrategias } \\
\text { orientadas a } \\
\text { desarrollar } \\
\text { de forma } \\
\text { óptima } \\
\text { procesos de } \\
\text { tránsito a la } \\
\text { edad adulta y } \\
\text { vida activa }\end{array}$ & \multicolumn{3}{|c|}{$\begin{array}{l}\text { Grupos de discusión con profesionales, familiares de personas } \\
\text { con DI y personas con DI. }\end{array}$} \\
\hline
\end{tabular}


Durante el segundo año (2013-2014), fueron doce los miembros del comité que accedieron a participar en una investigación sobre la vida independiente de las personas con discapacidad intelectual (tabla 3). Con el fin de ofrecerles la posibilidad de ejercer un rol más activo durante el desarrollo del estudio, se llevó a cabo un curso de formación en investigación educativa en el que participaron, de forma voluntaria, todos los miembros del comité asesor (Pallisera et al., 2014b; Puyalto et al 2015b). A lo largo del proceso se recogió información sobre las percepciones y puntos de vista de los miembros del comité asesor sobre su participación en dicha investigación. Los asesores consideraron la experiencia como una oportunidad de adquirir nuevas habilidades, de expresarse libremente sobre temas que les afectan y de implicarse en una actividad valiosa desde el punto de vista social (Puyalto, Pallisera, Fullana y Vilà, 2015a). 
Tabla 3: Objetivo y procedimiento de una investigación inclusiva sobre los procesos hacia la vida independiente de personas con discapacidad intelectual (DI)

\section{Título: Los procesos de apoyo a la vida independiente de las personas con discapacidad intelectual}

\begin{tabular}{|c|c|}
\hline Objetivo & Método \\
\hline $\begin{array}{l}\text { Identificar las } \\
\text { dificultades } \\
\text { a las que se } \\
\text { enfrentan }\end{array}$ & $\begin{array}{l}\text { Investigación inclusiva con un comité asesor en el que } \\
\text { participaron } 6 \text { personas que con discapacidad intelectual y cuatro } \\
\text { investigadoras sin discapacidad que dinamizaron las sesiones de } \\
\text { investigación mediante procedimientos de grupos de discusión, } \\
\text { con el apoyo de métodos visuales. }\end{array}$ \\
\hline
\end{tabular}

las personas

con DI para

concretar sus

proyectos

de vida

independiente

y los apoyos

que las ayudan

en diferentes

etapas de su

\begin{tabular}{l}
\hline Funciones \\
\hline Asesorar en el \\
planteamiento de \\
una investigación \\
sobre vida \\
independiente \\
a partir de la \\
exploración \\
de las propias \\
experiencias en el \\
desarrollo de sus \\
proyectos de vida. \\
\\
Realizar propuestas \\
para mejorar las \\
posibilidades de \\
poder desarrollar \\
los proyectos de \\
vida deseados.
\end{tabular}

Comité asesor

vida

\section{Procedimiento}

Se llevaron a cabo 7 reuniones con el comité asesor:

Reunión 1: Los asesores hablaron sobre sus experiencias en relación al tema de la vida independiente. Una investigadora recogió las aportaciones en una pizarra.

Reunión 2: A partir de fragmentos de películas y documentales sobre distintos tipos de vivienda los asesores discutieron sobre las distintas opciones.

Reunión 3: A partir de la presentación de casos los asesores formaron dos grupos de discusión en los que se debatió sobre uno de los casos y se puso en común.

Reunión 4: A partir de dibujos y fotografías realizadas por los mismos asesores y a través de un grupo focal se debatieron las barreras y los apoyos relacionados con la vida independiente.

Reunión 5: Se hicieron propuestas sobre las cosas que contribuyen al proyecto de vida independiente, se escribieron en la pizarra y ordenaron por importancia a través de un sistema de votación.

Reuniones 6 y 7: Los investigadores y los asesores trabajaron juntos en la presentación de la investigación para un curso de verano de la universidad. La conferencia se llevó a cabo de forma conjunta. 


\section{DEBATES EN TORNO A LA PARTICIPACIÓN DE PERSONAS CON DISCAPACIDAD INTELECTUAL EN LA INVESTIGACIÓN}

El desarrollo de la investigación inclusiva a lo largo de las dos últimas décadas no ha estado exento de debates. El impacto político y social que las investigaciones realizadas desde este enfoque puedan tener, más allá de su incidencia en el empoderamiento individual de sus participantes (García-Iriarte, O’Brien y Chadwich, 2014, Johnson, 2009, Johnson, Minogue y Hopkins, 2014, Stack y McDonald, 2014), o su contribución a la construcción de conocimiento (García-Iriarte et al 2014; Koenig, 2011), son algunos de los más relevantes que plantean dudas y retos sobre el sentido mismo de la investigación inclusiva. Sin embargo, en nuestro contexto, y desde nuestra experiencia en investigación inclusiva, creemos necesario hacer hincapié en algunos debates que se sitúan en el plano metodológico de este enfoque de investigación y que plantean retos y posibilidades para la inclusión de las personas con discapacidad intelectual en la investigación. Entre estos debates destacamos tres: la cuestión del apoyo que deben recibir las personas con discapacidad intelectual en la investigación, la representatividad de estas personas en las distintas fases de la investigación y la equidad en las relaciones entre investigadores con y sin discapacidad. A continuación abordamos cada uno de estos debates.

El apoyo a los investigadores con discapacidad intelectual para facilitar su participación en proyectos de investigación ha sido, desde el inicio de las experiencias de investigación inclusiva, uno de los debates más recurrentes. Algunos autores defienden que una de las formas de apoyar a las personas con discapacidad intelectual es ofreciéndoles formación en investigación y señalan que, según se incorporan de forma activa en distintas fases (diseño y aplicación de instrumentos, análisis de datos, difusión de los resultados, etc.) se hace más evidente la necesidad de dispongan de conocimientos sobre técnicas y procedimientos relacionados con estos procesos (Bigby y Frawley, 2010; Walmsley y Johnson, 2003; Walmsley, 2004; Ward y Simons, 1998).

Sin embargo no está claro qué tipo de formación deberían recibir ni el tipo de apoyos que habría que proporcionar. Strnadová et al. (2014) consideran que la formación en investigación es un importante elemento para implicar a personas con discapacidad intelectual en procesos de investigación. Proponen incidir en la comprensión del concepto de investigación, la determinación de cuáles son las cuestiones importantes que el equipo quiere responder, el acceso a los materiales escritos y la gestión de las tareas. No obstante, aunque existen experiencias de formación de investigadores con discapacidad intelectual (Cumming, Strnadová, Knox y Parmenter, 2014, Johnson, 2009; Salmon, Carey y Hunt, 2014) no existe un acuerdo sobre qué debería cubrir dicha formación, de modo que la preparación de esas personas es uno de los retos de la investigación inclusiva (Johnson, 2009, O’Brien et al., 2014).

El tema de la formación está estrechamente relacionado con otra cuestión que apenas ha sido abordada en la literatura sobre investigación inclusiva: el proceso de construcción del equipo de investigación. Se trata, sin duda, de un 
tema fundamental que se relaciona con la necesidad de constituir un equipo de trabajo capaz de llevar a cabo con éxito las distintas tareas de investigación. Para los investigadores con discapacidad, sentirse bien en el grupo durante todo el proceso de investigación es tan importante como encontrar una respuesta a sus preguntas. Por esta razón, Strnadová et al. (2014) plantean que el apoyo no únicamente tiene que ver con la formación en investigación, sino también con la cohesión del grupo que va a colaborar en ella.

Hasta qué punto el apoyo interfiere con la libertad de expresión y la toma de decisiones de las personas con discapacidad intelectual que participan en la investigación ha sido una cuestión ampliamente discutida (Kiernan, 1999, Williams et al., 2005). Con frecuencia, las publicaciones sobre investigaciones realizadas de forma inclusiva no concretan cómo se ha ofrecido ni en qué ha consistido el apoyo recibido por las personas con discapacidad intelectual (Bigby y Frawley, 2010). En este sentido, Walmsley (2004) propone que las investigaciones llevadas a cabo desde la perspectiva inclusiva expliquen quién ha hecho qué, cuál ha sido el rol desarrollado por los participantes en la investigación, y eviten que los roles de apoyo llevados a cabo por los investigadores sin discapacidad intelectual quede oculto. En este sentido, el desarrollo de instrumentos o la aplicación de algunas tecnologías para facilitar la accesibilidad de las personas con discapacidad intelectual a las distintas fases de la investigación ha sido objeto de algunos trabajos (Cumming et al., 2014, Kidney y McDonald, 2014).

Existe otro debate vinculado a la representatividad de las personas con discapacidad en la investigación. Como principio, la investigación inclusiva defiende que las personas con discapacidad intelectual deben poder estar representadas en los distintos momentos y fases de la investigación, desde la selección del tema a investigar hasta la construcción de conocimiento y la difusión de los resultados de la investigación. Ello implica un compromiso por parte de los investigadores sin discapacidad en considerar a las personas con discapacidad como informantes creíbles, capaces de generar sus propios significados, sin necesidad de triangulación ni de ejercer otro tipo de controles (Walmsley y Johnson, 2003). Algunos investigadores han cuestionado la posibilidad de que las personas con discapacidad intelectual estén representadas en todas las fases de la investigación. Se ha criticado su falta de rigor y el peligro de quedar atrapados en un ciclo de biografías sentimentales y anécdotas individuales. Se ha señalado también que la incorporación de personas con discapacidad intelectual a la investigación ha sido, con frecuencia, meramente simbólica o formal, sin corresponder a una real incorporación y representación de estas personas en las distintas fases de la investigación (Bigby et al., 2014b). De ahí que uno de los retos actuales de la investigación inclusiva tenga relación con cómo garantizar que las personas con discapacidad intelectual tengan un conocimiento real sobre cuál es su contribución al proceso. En este sentido la formación en investigación y el apoyo de los investigadores sin discapacidad van a ser elementos clave para facilitar el acceso a los materiales y a las tareas de investigación, y para trabajar en pro de la cohesión de grupo de forma que su 
participación sea verdaderamente relevante.

Una de las etapas más complejas en cualquier investigación es el análisis de la información, que debe conducir a la adquisición de nuevo conocimiento y que, según Nind (2011), presenta dificultades para los investigadores con discapacidad intelectual. La autora señala que probablemente no todos los aspectos del análisis de datos sean accesibles a estas personas. Para ella, no es tanto el análisis sino la interpretación de los datos, la reflexión sobre los resultados de la investigación, lo que puede llevarse a cabo de forma conjunta, contribuyendo así al avance del conocimiento. Stevenson (2014) muestra que los co-investigadores pueden adquirir habilidades de análisis de datos relacionadas con la codificación y la identificación de temas, si se les proporciona la formación adecuada.

Sin duda, es fácil que se produzca un desequilibrio de roles entre los investigadores con y sin discapacidad intelectual en algunas fases de la investigación, aunque no por ello se debe cuestionar el carácter inclusivo del conjunto de la investigación. Esta cuestión conecta con el tema de la equidad. El debate sobre la equidad implica una reflexión sobre las relaciones de poder que se establecen entre los miembros del equipo de investigación. Barton (2005) y Chappell (2000), reflexionando sobre la investigación emancipadora, y Dowse (2009), sobre la investigación colaborativa, enfatizan las dificultades para alcanzar un verdadero equilibrio de las relaciones de poder entre los investigadores con y sin discapacidad. Estos, y otros autores como McClimens et al. (2007), reconocen la complejidad de implementar los principios de la investigación inclusiva. La colaboración deseable entre investigadores con o sin discapacidad intelectual no es, a veces, el modo más eficiente de llevar a cabo investigaciones. Existen retos en relación a la comprensión de los objetivos de la investigación por parte de las personas con discapacidad intelectual y la comprensión de las implicaciones que tiene el dar su consentimiento, la toma de decisiones en las distintas fases del proceso y la difusión de los resultados.

Aunque los distintos enfoques de la investigación inclusiva parten de una relación respetuosa y un interés por las perspectivas de la personas con discapacidad intelectual, es necesario reconocer que este tipo de investigación no puede ser la solución universal para la generación de conocimiento sobre sus vidas, las políticas que les afectan o los servicios que utilizan (Bigby et al., 2014a). Las personas con discapacidad intelectual con necesidades de apoyo significativas tienen muchas más dificultades para dar a conocer sus necesidades básicas; por tanto, también va a ser difícil su participación en procesos de investigación. Hay que tener en cuenta, además, que las perspectivas de otras familiares, amigos, defensores o académicos sobre temas que afectan a las personas con discapacidad también tiene un valor importante. Algunos temas han sido identificados por otras personas y no por las mismas personas con discapacidad intelectual y ello no necesariamente resta relevancia a dichos temas.

La equidad también es difícil cuando nos enfrentamos a la tarea de difundir los resultados de la investigación. Las formas tradicionales de comunicar 
dichos resultados conllevan un alto dominio de habilidades de comprensión, pensamiento abstracto y comunicación escrita. Aunque los investigadores sin discapacidad intelectual realicen un borrador que pueda ser revisado por todos los participantes y aunque todos figuren como autores del documento, es evidente que las relaciones de poder son difíciles de equilibrar. Existen algunas publicaciones que admiten artículos en formato de lectura fácil y otras requieren resúmenes en dicho formato, con el objetivo de facilitar la difusión de resultados de investigaciones inclusivas y garantizar un mayor nivel de equidad. La mayoría de los artículos de lectura fácil se han publicado en la revista The British Journal of Learning Disabilities, siendo esta la única que acepta este tipo de artículos y constituye el medio principal de difusión de las investigaciones en las que han participado personas con discapacidad intelectual (Garbutt et al., 2010). También la Asociación Internacional para el Estudio Científico de las Discapacidades intelectuales y del desarrollo (IASSID) exige resúmenes en formato de lectura fácil en los congresos que organiza, y hay la posibilidad de realizar presentaciones accesibles a personas con discapacidad intelectual. Es otra vía de difusión aunque la participación de las personas con discapacidad resulta compleja puesto que requiere de pago de inscripciones, viajes, etc. El tema de la difusión es, pues, otro de los retos de la investigación inclusiva.

Otros aspectos relacionados en el debate de la equidad se refieren a la capacidad de las personas con discapacidad intelectual para dar consentimiento informado; ello implica comprender los riesgos y los beneficios de su participación. No se puede ignorar tampoco el hecho de que, a veces, las personas con discapacidad intelectual quedan excluidas porque existen muchas instituciones que velan por ellas y que temen que se les perjudique, o incluso que la propia institución sea evaluada en base a lo que explican estas personas en el marco de investigaciones inclusivas (McDonald y Kidney, 2012). Incluso los propios comités de ética se muestran a menudo reticentes a la incorporación de las personas con discapacidad en las investigaciones sin el consentimiento de terceras personas o instituciones, en aras a asegurar su protección. En definitiva, el debate sobre la equidad y las relaciones de poder es complejo. Por un lado, encontramos la voluntad de reconocer los derechos de las personas con discapacidad entre los cuales está el de autodeterminación y, por otro, el deseo de protección y de que estas personas no sufran abusos al participar en investigaciones. El equilibrio entre ambas posiciones es difícil.

Todos estos debates nos llevan a una pregunta final: toda la investigación sobre discapacidad ¿debería realizarse de forma inclusiva? Según Bigby et al. (2014a), la investigación inclusiva no está restringida a investigación en la que todos los participantes comparten poder y voz en igualdad de condiciones. Hasta la fecha, la mayoría de la investigación inclusiva se ha realizado con personas con discapacidad intelectual actuando más como co-investigadoras que como investigadoras que ejercen un completo control en el proceso de toma de decisiones sobre la investigación (Turk et al., 2012). Nind y Vinha (2014) alertan del riesgo de que la etiqueta de "investigación inclusiva" sea definitoria de una nueva "normalidad" dentro de un ámbito de investigación que podría quedar 
segregado por parte de la comunidad investigadora más amplia, perpetuando la situación contra la que investigadores y participantes han estado luchando. Tampoco puede convertirse en un dogma para la investigación en el campo de estudio sobre discapacidad (Strnadová y Cumming, 2014). Nind y Vinha (2014) defienden que es necesario ampliar la visión de la investigación inclusiva ya que entienden que no hay una única forma de hacer investigación inclusiva. Por ello, proponen hablar de "hacer investigación de forma inclusiva" más que de "hacer investigación inclusiva".

\section{RETOS Y PROPUESTAS METODOLÓGICAS A PARTIR DE UNA INVESTIGACIÓN DESARROLLADA INCLUSIVAMENTE}

La revisión bibliográfica realizada y nuestra propia experiencia en el desarrollo de una investigación desarrollada de forma inclusiva (Pallisera et al., 2013a, 2013c y 2014b, Puyalto et al., 2015a y 2015b) nos conducen a identificar algunos retos metodológicos importantes que tiene este tipo de investigación. Estos retos se relacionan con el propósito básico de la investigación inclusiva, que es conseguir la máxima participación de las personas con discapacidad intelectual en los procesos de investigación sobre temas que les afectan. Dicha participación implica tener oportunidades para participar, para expresarse con su propia voz y estar representados realmente en las distintas fases de la investigación. Asimismo, conlleva el compromiso de los investigadores para asegurar dicha participación, que incluye la facilitar la accesibilidad y evaluar de forma continua los procesos de investigación inclusiva. Comentamos, a continuación, estos temas.

Asegurar que las personas con discapacidad intelectual tengan oportunidades para participar en procesos de investigación que pueden resultar complejos no es tarea sencilla. Su participación implica, básicamente, que estas personas puedan expresarse con su propia voz en la investigación, por lo que resulta necesario utilizar métodos y procedimientos que ayuden a garantizar la libre expresión de los puntos de vista de las personas con discapacidad, que sus perspectivas se respeten y que su trabajo sea adecuadamente reconocido (Gill, 1999). Es conveniente utilizar métodos múltiples para garantizar la comprensión global de los fenómenos relacionados con la discapacidad. Además, las oportunidades para participar aumentan si se crean entornos en los que estas personas sientan confianza para expresarse. En este sentido, es necesario encontrar métodos que faciliten la expresión, estrategias diversas que puedan adaptarse a las características de las personas con discapacidad intelectual y que estén poco dirigidos por el investigador.

En la investigación que llevó a cabo nuestro equipo de investigación se optó por los grupos focales como estrategia para fomentar la participación de los miembros del comité asesor. El potencial de los grupos focales para favorecer la inclusión, la participación y el empoderamiento de las personas con discapacidad intelectual en la investigación ha sido señalada por Kaehne y O’Connell (2010), 
Cambridge y McCarty (2001) y Barr et al. (2010). Otra estrategia ha sido la utilización de métodos visuales como el fotovoz, método que consiste en que los participantes comenten libremente fotografías escogidas por ellos sobre las temáticas de análisis. La narración a partir de fotografías realizadas por los participantes en la investigación tiene un buen potencial para favorecer el carácter inclusivo de las investigaciones (Cumming et al., 2014; Fullana, Pallisera y Vilà, 2014).

Escuchar las voces de las personas con discapacidad intelectual no es el único elemento que garantiza su participación; se debe facilitar también que estas personas puedan participar de forma activa en las distintas fases de la investigación. Para lograrlo es necesario proporcionar información accesible a los participantes. En este sentido, uno de los retos de la investigación inclusiva es preparar materiales (presentaciones, consentimiento informado, trípticos informativos, etc.) con formatos de lectura fácil. En la investigación que realizamos se utilizaron distintas estrategias:

1. Las actas de las reuniones se redactaban en formato de lectura fácil, según las recomendaciones de la organización Change (2009).

2. Los materiales de trabajo se redactaban en lenguaje simplificado, y se acompañaban de dibujos o imágenes para facilitar su comprensión.

3. En las sesiones de trabajo había personal de apoyo perteneciente al mismo grupo de investigación, para ofrecer ayuda a las personas que lo requerían.

Las oportunidades de participar en la investigación aumentan si se ofrecen garantías de que las personas con discapacidad intelectual se sienten y están efectivamente representadas en las distintas etapas de la investigación. Desde nuestro punto de vista, los investigadores sin discapacidad intelectual deben tratar de encontrar fórmulas que promuevan esta representación en las distintas fases, bien sea como asesoras, como co-investigadoras o como líderes del proceso. Ello implica, por un lado, una revisión profunda de las relaciones entre los distintos miembros con y sin discapacidad intelectual del equipo de investigación, y una reflexión sobre el rol del investigador sin discapacidad intelectual. Por otro, significa un esfuerzo para asegurar que las personas con discapacidad intelectual pueden expresarse con voz propia y que sus perspectivas se respetan.

En cuanto al papel de los investigadores sin discapacidad intelectual, sea cual sea el papel que tengan las personas con discapacidad intelectual -asesoras, líderes o co-investigadoras- tienen que hacer un esfuerzo por actuar como facilitadores del proceso y encontrar fórmulas que permitan incorporar las perspectivas de las personas con discapacidad en la investigación, enfatizar el valor de sus aportaciones, y trabajar conjuntamente por la obtención de conocimiento. La investigación inclusiva supone un cambio del papel tradicional del investigador en la investigación sobre discapacidad y requiere que los investigadores sin discapacidad reflexionen desde el primer momento sobre su 
posición respecto a los demás implicados y sobre cómo esta puede influir en la investigación. Es necesario que hagan sus consideraciones sobre cuál es la voz que se va a escuchar a través de la investigación y a quién beneficia (Walsmley y Johnson, 2003) y que estén a disposición de las personas con discapacidad intelectual para poder atender sus necesidades, dudas y/o preocupaciones en torno al trabajo de investigación (Atkinson, 2005; Walmsley y Johnson, 2003). En nuestra investigación la relación entre investigadores con y sin discapacidad intelectual se desarrolló a partir de:

- La constitución de un comité asesor en la que las personas con discapacidad intelectual llevaron a cabo funciones de asesoría en distintos momentos de la investigación. Los investigadores sin discapacidad intelectual lideraban, en este caso, la investigación, pero se definieron unos momentos concretos en los que la intervención del comité asesor era relevante y se negociaba con sus miembros su participación.

- Se definieron con claridad las funciones del comité asesor en una serie de tareas de la investigación: la colaboración en la elaboración de una entrevista dirigida a jóvenes con discapacidad intelectual sobre su proceso de transición a la vida adulta, el análisis de datos procedentes de la entrevista, la participación en una dinámica de fotovoz para obtener sus puntos de vista sobre sus propios procesos de transición; y la participación en la elaboración de un póster que se presentó en la Conference in Disability Research. Nordic Network on Disability Research (NNDR), Turku, Finlandia, en mayo de 2013 (Pallisera et al., 2013b).

- Las investigadoras sin discapacidad intelectual participaron en las reuniones del comité asesor con distintas funciones: una investigadora moderó la discusión, otra realizó tareas de apoyo, recogiendo las ideas principales que iban surgiendo y anotándolas para poder disponer de ellas en las siguientes sesiones. Un tercer miembro del equipo recogió la información para poder elaborar el acta en formato de lectura fácil. Uno o dos miembros del equipo, según la sesión, realizaron funciones de apoyo individual cuando se estimaba necesario.

En el fondo, la accesibilidad conlleva desmitificar la investigación ofreciendo explicaciones claras y concisas de cada fase, centrarse en los elementos esenciales e importantes (O’Brien, McConkey y García-Iriarte, 2014).

Los retos de la participación, la representatividad y la equidad entre investigadores con y sin discapacidad solamente pueden afrontarse, a nuestro parecer, a través de la evaluación continua del propio proceso de investigación inclusiva. De este modo se puede obtener feedback de los investigadores con discapacidad intelectual que ayude a conocer si se sienten representados, respetados y si tienen oportunidades de participar. En nuestra investigación se llevó a cabo una evaluación continua a través de las siguientes estrategias 
dirigidas a recoger información de los miembros del comité asesor y de las investigadoras sin discapacidad intelectual.

- Un cuestionario inicial que recogía las primeras valoraciones de la experiencia expresadas por los participantes en el comité asesor, y las de las investigadoras sin discapacidad intelectual. Los temas incluían: los objetivos de las reuniones, la participación de los asesores, los roles de las investigadoras, las aportaciones hechas por los participantes, los materiales y apoyos utilizados y las propuestas de mejora.

- Grupos focales realizados con los participantes que permitieron fomentar la discusión y el debate entre los miembros del comité asesor, así como entre ellas y las investigadores, sobre los temas siguientes: objetivos de las reuniones, papel de las investigadores y accesibilidad de los materiales, los aprendizajes realizados durante el proceso de investigación, las implicaciones de participar en el comité asesor y propuestas de mejora.

- Entrevistas en grupo que sirvieron para explorar cuáles eran las opiniones y valoraciones de las investigadoras acerca de las reuniones en las que habían participado y, más concretamente, sobre los temas que se planteaban en los cuestionarios y en los grupos focales.

La investigación inclusiva con personas con discapacidad intelectual conlleva, por tanto, unos retos metodológicos que deben situarse más allá de la simple selección de unos u otros procedimientos o métodos. Como investigación participativa, con frecuencia con intención emancipadora, la investigación inclusiva supone reflexionar no solamente sobre aspectos técnicos de la investigación sino también sobre aspectos éticos, sobre las relaciones de poder entre investigadores y sobre la relación entre investigación y conocimiento científico en el campo de la investigación sobre discapacidad y entre investigación, prácticas y políticas.

Nuestra experiencia teórico-práctica en el campo de la investigación inclusiva nos lleva a considerar que los grupos de investigación tienen un papel fundamental en la incentivación del desarrollo de investigaciones que se llevan a cabo, de forma inclusiva, como estrategia dirigida a empoderar a las personas con discapacidad intelectual para la defensa de sus derechos, incidiendo, al fin y al cabo, en la transformación de las políticas y de las prácticas que les afectan. Sin embargo, no puede obviarse que el avance de la investigación inclusiva está estrechamente relacionado con la transformación de los servicios que trabajan con personas con discapacidad intelectual. En la medida en que estas personas sean efectivamente las protagonistas de las decisiones que afectan a sus vidas y se conviertan en el centro de las acciones de apoyo, dispondrán de instrumentos más efectivos para reivindicar un papel activo en la investigación. Por ello, el compromiso de los investigadores en la investigación inclusiva va más allá de la reflexión y transformación del modo de investigar. El trabajo colaborativo con los profesionales de los servicios que atienden y apoyan a estas personas 
constituye, también, una base para contribuir a que los servicios y proyectos socioeducativos organicen los apoyos basándose realmente en las necesidades, demandas y proyectos personales de las personas con discapacidad intelectual.

Dar a conocer los resultados de estos trabajos es esencial para poder incidir en la transformación de las prácticas. Sin embargo, los investigadores, con frecuencia, no disponen de los apoyos ni incluso de los foros adecuados para que su participación pueda ir más allá del mero simbolismo. En este sentido, uno de los retos de la investigación inclusiva es desarrollar espacios de difusión que no constituyan, como ocurre de facto la mayoría de las ocasiones, una vía restringida a académicos y profesionales

\section{BIBLIOGRAFÍA}

ABELL, S., ASHMORE, J.; BEART, S.; BROWNLEY, P.; BUTCHER, A.; CLARKE, Z.; COMBES, H.; FRANCIS, E.; HAYES, S.; HEMMINGHAM, I.; HICKS, K.; IBRAHAM, A.; KENYON, E.; LEE, D.; MCCLIMENS, A.; COLLINS, M.; NEWTON, J. \& WILSON, D. (2007). "Including everyone in research: The Burton Street Research Group", British Journal of Learning Disabilities, 35, 121-124.

ABBOT, S. \& MCCONKEY, R. (2006). "The barriers to social inclusion as perceived by people with intellectual disabilities", Journal of Intellectual Disabilities, 20, 3: 275-287.

ATKINSON, D. (2005). "Research as Social Work: Participatory Research in Learning Disability", British Journal of Social Work, 35, 425-234.

BARR, O., MCCONKEY, R. \& MCCONAGUIE, J. (2010). "Views of People with Learning Difficulties about Current and Future Accommodation: The use of focus groups to promote discussion", Disability \& Society, 18, 5: 577-597.

BARTON, L. (2005). "Emancipatory research and disable people: some observations and questions", Educational Review, 57, 3: 317-327.

BIGBY, C. \& FRAWLEY, P. (2010). "Reflections on doing inclusive research in the "Making Life Good in the Community study", Journal of Intellectual Developmental Disability, 35, 2:. 53-61.

BIGBY, C., FRAWLEY, P. \& RAMCHARAN, P. (2014a) "Conceptualizing Inclusive Research with People with Intellectual Disability", Journal of Applied Research in Intellectual Disabilities, 27, 3-12.

BIGBY, C., FRAWLEY, P. \& RAMCHARAN, P. (2014b). "A Collaborative Group Method of Inclusive Research", Journal of Applied Research in Intellectual Disabilities, 27, 54-64.

BERESFORD, B. (2004). "On the road to nowhere? Young disabled people and transition", Child: Care, Health \& Development, 30, 6: 581-587.

BOLAND, M.; DALY, L., \& STAINES, A. (2008). "Methodological issues in inclusive intellectual disability research: A Health promotion needs assessment of people attending Irish disability Services". Journal of Applied Research in Intellectual Disabilities, 21, 199-209

BROOKS, M. \& DAVIES, S. (2008). "Pathways to participatory research in developing a tool to measure feelings", British Journal of learning Disabilities, 36, 128-133. 
CAMBRIDGE, P. \& MCCARTHY, M. (2001). "User focus groups and Best Value in services for people with learning disabilities", Health and Social Care in the Community, 9, 476-489.

CHAPPELL, A. L. (2000). "Emergence of participatory methodology in learning difficulty research: unterstanding the context". British Jorunal of Learning Disabilities, 28, 28-43.

CLARKE, S., SLOPER, P., MORAN, N.,CUSWORTH, L., FRANKLIN, A. \& BEECHAM, J. (2011). "Multi-agency transition services: greater collaboration needed to meet the priorities of Young people with complex needs as they move into adulthood", Journal of Integrated Care, 19, 5: 30-40.

CHANGE (2009). How to make information accessible. A guide to producing easy read documents, en http://www.changepeople.co.uk (consulta: 15 de junio de 2012).

CORTÉS, S. (2012). "Modelo de participación para personas con discapacidad intelectual en APADIS 'Nosotros también investigamos"', IV Premio Investigación e innovación sobre personas con Discapacidad Intelectual. Trabajos premiados. Ed. AMPANS, 190-276 en http://www.ampans.cat (consulta: 10 de julio de 2013).

CUMMING, T.M., STRNADOVÁ, I., KNOX, M. \& PARMENTER, T. (2014). "Mobile Technology in inclusive research: tools of empowerment". Disability \& Society, 29, 7: 999-1012

DEGUARA, M., JElASSI, O., MICALlEF, B. \& CALLUS, A.M. (2012), "How we like to live when we have the chance", British Journal of Learning Disabilities, 40, 123-127.

DOWSE, L. (2009). "'I'ts like being in a zoo.' Reearching whith people with intellectual disability", Journal of Research in Special Educational Needs, 9, 3: 141-153.

EMERSON, E. \& RAMCHARAN, P. (2010). "Models of service delivery", en G. GRANT, P. RAMCHARAN, M. FLYNN \& M. RICHARDSON (eds.) Learning Disability: A Life Cycle Approach (2nd Ed). Berkshire, Open University Press, pp. 59-72.

EUROPEAN COMMISSION (2009). Report of the Ad Hoc Expert Group on the Transition from Institutional to Community-based Care. European Communities.

FRA- EUROPEAN UNION AGENCY FOR FUNDAMENTAL RIGHTS (FRA) (2013). Choice and control: the right to independent living. Experiences of persons with intellectual disabilities and persons with mental health problems in nine EU Member States. Luxembourg, Publications Office of the European Union.

FULLANA, J., PALLISERA, M. y VILÀ, M. (2014). "Advancing towards inclusive social research: visual methods as opportunities for people with severe mental illness to participate in research", International Journal of Social Research Methodology 17:6, 723-738 http://dx.doi.org/10.1080/13645579.2013.832049

GARBUTT, R., TATTERSALL, J., DUNN, J. \& BOYCOTT-GAMETT, R (2010). "Accessible article: involving people with learning disabilities in Research", British Journal of Learning Disabilities, 38, 21-34.

GARCÍA-IRIARTE, E.; O'BRIEN, P.; CHADWICH, D. (2014). “Invonving people with intellectual disabilities within research teams: lessons learned from an Irish experience". Journal of Policy and Practice in Intellectual Disabilities, 11, 2: 149157.

GILL, C. J. (1999). "Invisible ubiquity: The surprising relevance of disability issues in evaluation", American Journal of Evaluation, 20, 279-297.

GONZÁLEZ LUNA, B. (2013). "Investigando con personas con dificultades de aprendizaje". Revista Española de Discapacidad, 1, 2: 77-94. 
HAIGH, A.; LEE, D.; SHAW, C.; HAWTHORNE, M.; CAMBERLAIN, S.; NEWMAN, D.W., CLARKE, Z, \& BEAIL, N. (2013). "What Thincs make people with a learning Disability Happy and Satisfied with their lives: an inclusive research project". Journal of Applied Research in Intellectual Disabilities, 26, 26-33

HAYA, I., ROJAS, S., LAZARO, S. (2014). "Observaciones metodológicas sobre la investigación inclusiva: 'Me gustaría que sacarais que la persona con discapacidad tiene su propio pensamiento"”. Revista de investigación en educación, 12, 2:135144.

JOHNSON, K. (2009). "No longer researching about us without us: a researcher's reflection on rights and inclusive research in Ireland". British Journal of Learning Disabilities, 37, 250-256

JOHNSON, K., MINOGUE, G.; HOPKINS, R. (2014). "Inclusive Research: Making a Difference to Policy and Legislation". Journal of Applied Research in Intellectual Disabilities, 27, 76-84

KAEHNE, A. \& O'Connell, C. (2010). "Focus groups with people with learning disabilities", Journal of Intellectual Disabilities, 14, 2: 133-145.

KEYES, S.E.; BRANDON, T. (2011). "Mutual Support: a model of participatory suport by and for people with learning difficulties". British Journal of Learning Disabilities, 40, 222-228.

KIDNEY, C.A.; MCDONALD, K.E. (2014). “A Toolkit for accessible and respectful engagement in research". Disability \& Society, 27, 7: 1013-1030

KIERNAN, C. (1999). "Participation in research by people with learning disability: origins and issues", British Journal of Learning Disabilities, 27, 43-47.

KOENIG, O. (2011). "Any added value? Co-constructing life stories of and with people with intellectual disabilities". British Journal of Learning Disabilities, 40, 213-221

McCLIMENS, A., Grant, G. \& Ramcharan, P. (2007., "Looking in a fairground mirror: Reflections on partnerships in learning disability research" en M. NOLAN, E. HANSON, G. GRANT \& J. KEADY (eds.), User participation in health and social care research, Berkshire, McGraw Hill and Open University Press, 104-119.

McCONKEY, R., Sowney, M., Milligan, V. \& Barr, O (2004). "Views of people with Intellectual Disabilities of Their Present and Future Living Arrangements", Journal of Policy and Practice in Intellectual disabilities. 1, 3/4: 113-125.

McDONALD, K. E. \& KIDNEY, C.A. (2012). "What is right? Ethics in Intellectual Disabilities Research", Journal of Policy and Practice in Intellectual Disabilities, 9, 27-39.

MICHELL, B. (2012). “Checking Up On Des: My Life My Choice's research into annual health checks for people with learning disabilities in Oxfordshire", British Journal of Learning Disabilities, 40, 152-161.

NACIONES UNIDAS (2006). Convención de las Naciones Unidas sobre los derechos de las personas con discapacidad, disponible en http://www.un.org/spanish/ disabilities [consulta: 28 enero 2013]

NIND, M. (2011). "Participatory data analysis: a step too far?", Qualitative Research, 11, 4: 349-363.

NIND, M. \& VINHA, H. (2014). "Doing research inclusively: bridges to multiple possibilities in inclusive research", British Journal of Learning Disabilities, 42, 2 : 102-109.

O'BRIEN, P.; MCCONKEY, R.; GARCÍA-IRIARTE, E. (2014). "Co-researching with people who have intellectual disabilities: Insights from a National Survey”. Journal of Applied Research in Intellectual Disabilities, 27, 65-75. 
OLLERTON, J.; HORSFALL, D. (2013). "Rights to research: utilising the Convention on the Rights of persons with disabilities as an inclusive participatory action research tool". Disability \& Society, 28 5: 616-630.

PALLISERA, M., MARTÍN, R., PUYALTO, C., FULLANA, J., VILÀ, M., JIMÉNEZ, P. y CASTRO, M. (2013a), “Cómo apoya la escuela el proceso de transición a la vida adulta? Experiencias de jóvenes con discapacidad intelectual en el contexto de una investigación inclusiva". X Congreso Internacional. XX Jornadas de Universidades y Educación Especial. Educación Inclusiva. Desafios y respuestas creativas. Universidad de Zaragoza. Comunicación, disponible en http://www. unizar.es/educacioninclusivacreativa/sites/default/files/Actas.pdf

PALLISERA, M., PUYALTÓ, C., MARTÍN, R., VILÀ, M., FULLANA, J., BAGUÉ, A., BATLLE, J., COLOMER, A., ESPINOSA, M., MARTÍ, P., MARTÍNEZ, M., RIU, L., RUIZ, F. Y SAUBÍ, J. (2013b, mayo) "What do we have learnt doing inclusive research? A research on the transition to adulthood". Póster presentado en la Conference in Disability Research. Nordic Network on Disability Research (NNDR), Turku, Finlandia.

PALLISERA, M.; FULLANA, J.; VILÀ, M.; PUYALTO, C.; MARTÍN, R. (2013c). "La transición a la edad adulta y vida activa de jóvenes con discapacidad intelectual: análisis y propuestas a partir de una investigación inclusiva”. Investigación de Calidad para Mejorar la Educación. II Congreso Internacional Multidisciplinar de Investigación educativa, 706-711.

PALliSERA, M., VILÀ, M., FULlANA, J., MARTÍN, R., PUYALTÓ, C. (2014a) “¿Continuidad o fragmentación? Percepción de los profesionales sobre la coordinación entre servicios en los procesos de tránsito a la vida adulta de los jóvenes con discapacidad intelectual". Educatio Siglo XXI, 32, 2: 213-232.

PALlisera, M., PUYAltó, C., FUllanA, J., VIlÂ, M. Y CASTRO, M. (2014b). "'Investigamos junt@s': Un curso de formación en investigación educativa para personas con discapacidad intelectual". Comunicación presentada en el XI Congreso Internacional y XXXI Jornadas de Universidades y Educación Inclusiva, Castellón, España, disponible en http://www.quadernsdigitals.net/index. php?accionMenu=hemeroteca. VisualizaArticuloIU.visualiza\&articulo_id=11297

PUYALTO, C.; PALLISERA, M.; FULLANA, J.; VILÀ, M. (2015a). "Doing Research Together: A Study on the Views of Advisors with Intellectual Disabilities and Non Disabled Researchers Collaborating in Research". Journal of Applied Research in Intellectual Disabilities. DOI: 10.1111/jar.12165

PUYALTO, C.; PALLISERA, M.; FULLANA, J.; VILÀ, M.; CASTRO, M. (2015b). "Investigando con personas con discapacidad en la universidad: una experiencia de colaboración entre investigadores y asesores con discapacidad intel-lectual". IX Jornadas Científicas Internacionales de Investigación sobre Personas con Discapacidad. Prácticas profesionales y Organizaciones Basadas en la Evidencia (1-10). Salamanca: INICO

SALMON, N., CAREY, E., HUNT, A. (2014). "Research Skills for People with Intellectual Disabilities", Learning Disability Practice, 17, 3: 27-35

STACK, E.; MCDONALD, K. (2014). "Nothing about us without us: Does action research in Developmental disabilities research measure up?" Journal of Policy and practice in Intellectual Disabilities, 11, 2: 83-91

STEVENSON, M- (2014). "Participatory Data Analysis alongside co-researchers who have Down Syndrome”. Journal of Applied Research in Intellectual Disabilities, $27,23-33$ 
STRNADOVÁ, I.; \& CUMMING, T. M. (2014). "Editorial. People with intellectual disabilities conducting research: new directions for inclusive research". Journal of Applied Research in Intellectual Disabilities, 27, 1-2

STRNADOVÁ, I., CUMMING, T., KNOX, M., PARMENTER, T. \& WELCOME TO OUR CLASS RESEARCH GROUP (2014). "Building an Inclusive Research Team: The importance of team building and skills training", Journal of Applied Research in Intellectual Disabilities, 27, 13-22.

SUSINOS, T.; PARRILLA, A. (2008). "Dar la voz en la investigación inclusiva. Debates sobre inclusión y exclusión desde un enfoque biográfico-narrativo". Revista Electrónica Iberoamericana sobre Calidad, Eficacia y Cambio en Educación, 6, 2: 157-171.

SUSINOS, T.; PARRILLA, A. (2013). "Investigación inclusiva en tiempos difíciles. Certezas provisionales y debates pendientes”. Revista Electrónica Iberoamericana sobre Calidad, Eficacia y Cambio en Educación, 11, 2: 88-98.

TUFFREY-WIJNE, I. \& BUTLER, G. (2009). "Co-researching with people with learning disabilities: an experience of involvement in qualitative data analysis", Health Expectations, 13, 174-184.

TURK, V., LEER, G., BURCHELL, S., KHATTRAM, S., CORNEY, R. \& Rowlands, G. (2012),.“Adults with Intellectual Disabilities and their Carers as Researchers and Participants in a RCT". Journal of Applied Research in Intellectual Disabilities, 25, $1-10$.

WALMSLEY, J. (2001). "Normalisation, emancipatory research and inclusive research in learning disability", Disability \& Society, 16: 187-205.

WALMSLEY, J. (2004). "Involving users with learning difficulties in Health improvement: lessons from inclusive learning disability research", Nursing Inquiry. 11, 54-64.

WALMSLEY, J. \& THE CENTRAL ENGLAND PEOPLE FIRST HISTORY PROJECT TEAM (2014). "Telling the History of Self-Advocacy: A Challenge for Inclusive Research", Journal of Applied Research in Intellectual Disabilities, 27, 34-43.

WALMSLEY, J. \& Johnson, K. (2003). Inclusive Research with People with Learning Disabilities: Past, Present and Future, London, Jessica Kingsley Publishers.

WARD, L. \& Simons, K. (1998). "Practising Partnership: Involving People with Learning Difficulties in Research", British Journal of Learning Disabilities. 26, 128-131.

WHITE, E. L. \& MORGAN, M.F. (2012). "Yes! I am a researcher. The research story of a young adult with Down Syndrome". British Journal of Learning Disabilities, 40, 101-108.

WILLIAMS, P. \& SHOULTZ, B. (1982). We can speak for ourselves. Human Horizon Series. London: Souvenir Press.

WILLIAMS, V., SIMONS, K. \& SWINDON PEOPLE FIRST RESEARCH TEAM (2005) ."More researching together: The role of nondisabled researchers in working with People First Members", British Journal of Learning Disabilities, 33, 6-14.

YOUNG, A. F. \& Chesson, R.A. (2007). "Determining research questions on Health risks by people with learning disabilities, carers and care-workers", British Journal of Learning Disabilities, 36, 22-31. 